\title{
ROTATIONALLY INVARIANT PERIODIC SOLUTIONS OF SEMILINEAR WAVE EQUATIONS
}

\author{
MARTIN SCHECHTER*
}

\begin{abstract}
Under suitable conditions we are able to solve the semilinear wave equation in any dimension. We are also able to compute the essential spectrum of the linear wave operator for the rotationally invariant periodic case.
\end{abstract}

\section{INTRODUCTION}

In this paper we continue the work of Smiley [SM] and Ben-Naoum and Mawhin [BNM1] concerning radially symmetric solutions for the problem

$$
\begin{gathered}
u_{t t}-\Delta u=f(t, x, u), \quad t \in \mathbf{R}, \quad x \in B_{R} \\
u(t, x)=0, \quad t \in \mathbf{R}, \quad x \in \partial B_{R} \\
u(t+T, x)=u(t, x), \quad t \in \mathbf{R}, \quad x \in B_{R},
\end{gathered}
$$

where

$$
B_{R}=\left\{x \in \mathbf{R}^{n}:|x|<R\right\} .
$$

Our basic assumption is that

$$
8 R / T=a / b
$$

where $a, b$ are relatively prime positive integers. We show that

$$
n \not \equiv 3 \quad(\bmod (4, a))
$$

1991 Mathematics Subject Classification. Primary: 35B10, 35L05, 35P20, 47H15, 49J35, 58E05.

Key words and phrases. Critical point theory, variational methods, saddle point theory, semilinear differential equations.

* Research supported in part by a NSF grant.

Received: February 11, 1998. 
implies that the linear problem corresponding to (1.1) - (1.3) has no essential spectrum. If

$$
n \equiv 3 \quad(\bmod (4, a)),
$$

then the essential spectrum of the linear operator consists of precisely one point

$$
\lambda_{0}=-(n-3)(n-1) / 4 R^{2} .
$$

(This shows that the spectrum has at most one limit point.) We can then consider the nonlinear case

$$
f(t, r, s)=\mu s+p(t, r, s),
$$

where $\mu$ is a point in the resolvent set, $r=|x|$, and

$$
|p(t, r, s)| \leq\left(|s|^{\theta}+1\right), \quad s \in \mathbf{R}
$$

for some number $\theta<1$. Our main theorem is

Theorem 1.1. If (1.5) holds, then (1.1) - (1.3) has a weak rotationally invariant solution. If (1.6) holds and $\lambda_{0}<\mu$, assume in addition that $p(t, r, s)$ is nondecreasing in $s$. If $\mu<\lambda_{0}$, assume that $p(t, r, s)$ is nonincreasing in $s$. Then (1.1) - (1.3) has a weak rotationally invariant solution.

The case $\mathrm{T}=2 \pi, 2 R=\pi$ was considered in detail in [BNM1]. They proved the existence of a weak solution for $n$ even and $n=1,3$. They consider more general situations than (1.8), (1.9). However, our methods can be adjusted to cover their case as well. Uniqueness theorems were also treated in [BNM1]. They also considered odd $n>5$ when the spectrum of the linear problem is not dense. However, they do not establish when this is the case.

A main consideration in our approach is the following theorem concerning infinite dimensional linking. It is of interest in its own right and has several other applications.

Theorem 1.2. Let $N$ be a closed separable subspace of a Hilbert space E. Let $G$ be a continuously differentiable functional on $E$ such that

$$
v_{n}=P u_{n} \rightarrow v \text { weakly in } E, \quad w_{n}=(I-P) u_{n} \rightarrow \text { w strongly in } E
$$
implies

$$
G^{\prime}\left(v_{n}+w_{n}\right) \rightarrow G^{\prime}(v+w) \text { weakly in } E,
$$

where $P$ is the projection of $E$ onto $N$. Let $Q$ be a bounded open convex subset of $N$, and let $F$ be a continuous map of $E$ onto $N$ such that

$$
\left.F\right|_{N}=I, F(v-w)=v-F w, \quad v \in N, w \in E .
$$

Assume

$$
a_{0}:=\sup _{A} G \leq b_{0}:=\inf _{B} G, a_{1}:=\sup _{Q} G<\infty,
$$

where $A=\partial Q, B=F^{-1}(p)$ and $p$ is a point in $Q$. Then there is a sequence $\left\{u_{k}\right\} \subset E$ such that

$$
G\left(u_{k}\right) \rightarrow c, b_{0} \leq c \leq a_{1}, G^{\prime}\left(u_{k}\right) \rightarrow 0 .
$$


Theorem 1.2 will be proved in Section 4. It generalizes theorems in $[\mathrm{KS}, \mathrm{S} 2,3, \mathrm{Wi}$. Theorem 1.1 will be proved in Section 3 after the essential spectrum of the linear operator is determined in Section 2.

\section{The Spectrum of the Linear operator}

In proving Theorem 1.1 we shall need to calculate the spectrum of the linear operator $\square$ applied to periodic rotationally symmetric functions. Specifically, we shall need

Theorem 2.1. Let $L_{0}$ be the operator

$$
L_{0} u=u_{t t}-u_{r r}-r^{-1}(n-1) u_{r}
$$

applied to functions $u(t, r)$ in $C^{\infty}(\bar{\Omega})$ satisfying

$$
\begin{gathered}
u(T, r)=u(0, r), u_{t}(T, r)=u_{t}(0, r), \quad 0 \leq r \leq R \\
u(t, R)=u_{R}(t, 0)=0, \quad t \in \mathbf{R},
\end{gathered}
$$

where $\Omega=[0, T] \times[0, R]$. Then $L_{0}$ is symmetric on $L^{2}(\Omega, \rho)$, where $\rho=$ $r^{n-1}$. Assume that $8 R / T=a / b$, where $a, b$ are relatively prime integers (i.e., $(a, b)=1)$. Then $L_{0}$ has a selfadjoint extension $L$ having no essential spectrum other than the point $\lambda_{0}=-(n-3)(n-1) / 4 R^{2}$. If $n \not \equiv 3(\bmod (4, a))$, then $L$ has no essential spectrum. If $n \equiv 3(\bmod (4, a))$, then the essential spectrum of $L$ is precisely the point $\lambda_{0}$.

Proof. Let $\nu=(n-2) / 2$, and let $\gamma$ be a positive root of $J_{\nu}(x)=0$, where $J_{\nu}$ is the Bessel function of the first kind. Set

$$
\varphi(r)=J_{\nu}(\gamma r / R) / r^{\nu}
$$

Then

$$
\varphi^{\prime \prime}+(n-1) \varphi^{\prime} / r=\left(x^{2} J_{\nu}^{\prime \prime}+x J_{\nu}^{\prime}-\nu^{2} J_{\nu}\right) / r^{\nu+2}=-\gamma^{2} J_{\nu} / R^{2} .
$$

If

$$
\psi(t, r)=\varphi(r) e^{2 \pi i k t / T},
$$

then

$$
L_{0} \psi=\left[(\gamma / R)^{2}-(2 \pi k / T)^{2}\right] \psi
$$

Let $\gamma_{j}$ be the $j$-th positive root of $J_{\nu}(x)=0$, and set

$$
\psi_{j k}(t, r)=r^{-\nu} J_{\nu}\left(\gamma_{j} r / R\right) e^{2 \pi i k t / T} .
$$

Then $\psi_{j k}(t, r)$ is an eigenfunction of $L_{0}$ with eigenvalue

$$
\lambda_{j k}=\left(\gamma_{j} / R\right)^{2}-(2 \pi k / T)^{2} .
$$

It is easily checked that the functions $\psi_{j k}$, when normalized, form a complete orthonormal sequence in $L^{2}(\Omega, \rho)$. We shall show that the corresponding eigenvalues (2.7) are not dense in $\mathbf{R}$. It will then follow that $L_{0}$ has a selfadjoint extension $L$ with spectrum equal to the closure of the set $\left\{\lambda_{j k}\right\}$. Now

$$
\gamma_{j}=\beta_{j}-(\mu-1) / 8 \beta_{j}+O\left(\beta_{j}^{-3}\right) \text { as } \beta_{j} \rightarrow \infty,
$$


where

$$
\beta_{j}=\pi\left(j+\frac{1}{2} \nu-\frac{1}{4}\right), \quad \mu=4 \nu^{2}
$$

(cf., e.g., [WA]). Thus

$$
\begin{aligned}
\lambda_{j k} R^{2}= & {\left[\beta_{j}-\tau_{k}-(\mu-1) / 8 \beta_{j}+O\left(\beta_{j}^{-3}\right)\right] } \\
& \cdot\left[\beta_{j}+\tau_{k}-(\mu-1) / 8 \beta_{j}+O\left(\beta_{j}^{-3}\right)\right] \\
= & \beta_{j}^{2}-\tau_{k}^{2}-(\mu-1) / 4+O\left(\beta_{j}^{-2}\right),
\end{aligned}
$$

where $\tau_{k}=2 k \pi R / T$. (We may assume $k \geq 0$.) Now

$$
\beta_{j}-\tau_{k}=\pi\left(j+\frac{1}{2} \nu-\frac{1}{4}-a k / 4 b\right)=\pi[(4 j+n-3) b-a k] / 4 b .
$$

Since the expression in the brackets is an integer, we see that either $\beta_{j}=\tau_{k}$ or

$$
\left|\beta_{j}-\tau_{k}\right| \geq \pi / 4 b
$$

Thus

$$
\lim _{\substack{j,|k| \rightarrow \infty \\ \beta_{j}=\tau_{k}}} \lambda_{j k}=-(\mu-1) / 4 R^{2}=\lambda_{0}
$$

and

$$
\lim _{\substack{j,|k| \rightarrow \infty \\ \beta_{j} \neq \tau_{k}}}\left|\lambda_{j k}\right|=\infty
$$

If $n-3$ is not a multiple of $(4, a)$, then

$$
\beta_{j}-\tau_{k}=\pi(4 j+n-3-a k / b) / 4
$$

can never vanish. To see this, note that if $(b, k) \neq b$, then $a k / b$ is not an integer. Hence $\beta_{j} \neq \tau_{k}$. If $b=(b, k)$, then

$$
(n-3) \neq a k^{\prime}-4 j \quad \forall j, k^{\prime}=k / b .
$$

Thus in this case we always have $\beta_{j} \neq \tau_{k}$ and $\left|\lambda_{j k}\right| \rightarrow \infty$ as $j, k \rightarrow \infty$. On the other hand, if $n \equiv 3(\bmod (4, a))$, then there is an infinite number of positive integers $j, k^{\prime}$ such that

$$
n-3=a k^{\prime}-4 j .
$$

Hence, the point $\lambda_{0}$ is a limit point of eigenvalues. Consequently, it is in $\sigma_{e}(L)$. This completes the proof.

\section{The Nonlinear Case}

We now turn to the problem of solving

$$
L u=f(t, r, u), \quad u \in D(L),
$$

where $L$ is the selfadjoint extension of the operator $L_{0}$ given in Theorem 2.1. Under the hypotheses of that theorem the spectrum of $L$ is discrete. We assume that

$$
f(t, r, s)=\mu s+p(t, r, s),
$$


where $\mu$ is a point in the resolvent set of $L$ and $p(t, r, s)$ is a Carathéodory function on $\Omega \times \mathbf{R}$ such that

$$
|p(t, r, s)| \leq c\left(|s|^{\theta}+1\right), \quad s \in \mathbf{R}
$$

for some number $\theta<1$. We have

Theorem 3.1. Let $f(t, r, s)$ satisfy (3.2) and (3.3), and assume the hypotheses of Theorem 2.1. If

$$
n \not \equiv 3(\bmod (4, a)),
$$

make no further assumptions. If

$$
n \equiv 3(\bmod (4, a))
$$

and $\lambda_{0}<\mu$, assume that $p(t, r, s)$ is nondecreasing in $s$. If (3.5) holds and $\mu<\lambda_{0}$, assume that $p(t, r, s)$ is nonincreasing in $s$. Then (3.1) has at least one weak solution.

Proof. Since $\mu$ is in the resolvent set of $L$, there is a $\delta>0$ such that

$$
\left|\lambda_{j k}-\mu\right| \geq \delta \quad \forall j, k
$$

where the $\lambda_{j k}$ are given by (2.7). Each $u \in L^{2}(\Omega, \rho)$ can be expanded in the form

$$
u=\sum \alpha_{j k} \psi_{j k}(t, r),
$$

where the $\psi_{j k}$ are given by (2.6). Let $N_{0}$ be the subspace of those $u \in$ $L^{2}(\Omega, \rho)$ for which $\alpha_{j k}=0$ if $\beta_{j} \neq \tau_{k}$ (cf. the proof of Theorem 2.1). For $u \in N_{0}$

$$
u=\sum_{[0]} \alpha_{j k} \psi_{j k}(t, r),
$$

where summation is taken over those $j, k$ for which $\beta_{j}=\tau_{k}$. Let $E$ be the subspace of $L^{2}(\Omega, \rho)$ consisting of those $u$ for which

$$
\|u\|_{E}^{2}=\sum\left|\lambda_{j k}-\mu \| \alpha_{j k}\right|^{2}
$$

is finite. With this norm, $E$ becomes a separable Hilbert space. Note that $E \subset D\left(|L|^{1 / 2}\right)$, and the embedding of $E \ominus N_{0}$ into $L^{2}(\Omega, \rho)$ is compact (we use (2.13) for this purpose). Let

$$
G(u)=([L-\mu] u, w)-2 \iint_{\Omega} P(t, r, u) \rho d t d r, \quad u \in E,
$$

where

$$
P(t, r, s)=\int_{0}^{s} p(t, r, \sigma) d \sigma,
$$

and the scalar product is that of $L^{2}(\Omega, \rho)$. One checks readily that $G$ is a $C^{1}$ functional on $E$ with

$$
\left(G^{\prime}(u), v\right) / 2=([L-\mu] u, v)-(p(u), v), \quad u, v \in E,
$$

where we write $p(u)$ in place of $p(t, r, u)$. This shows that $u$ is a weak solution of (3.1) iff $G^{\prime}(u)=0$. 
Let $N$ be the subspace of $E$ spanned by the $\psi_{j k}$ corresponding to those $\lambda_{j k}<\mu$ and let $M$ denote the subspace of $E$ spanned by the rest. Thus $M=N^{\perp}$ in $E$. Assume first that $N \cap N_{0}=\{0\}$. Then

$$
\|u\|_{E}^{2}=\sum\left(\mu-\lambda_{j k}\right)\left|\alpha_{j k}\right|^{2}, \quad u \in N .
$$

Thus

$$
\begin{aligned}
G(v) & =-\|v\|_{E}^{2}-2 \iint_{\Omega} P(t, r, v) \rho d t d r \\
& \leq-\|v\|_{E}^{2}+C \iint_{\Omega}\left(|v|^{1+\theta}+|v|\right) \rho d t d r \\
& \leq-\|v\|_{E}^{2}+C^{\prime}\left(\|v\|^{1+\theta}+\|v\|\right) \rightarrow-\infty, \quad\|v\|_{E} \rightarrow \infty, \quad v \in N .
\end{aligned}
$$

If $w \in M$,

$$
G(w) \geq \delta\|w\|^{2}-C\left(\|w\|^{1+\theta}+\|w\|\right) \geq-K, \quad w \in M .
$$

We can now make use of Theorem 1.2. If $Q$ is a large ball in $N$, then

$$
\sup _{\partial Q} G \leq \inf _{M} G
$$

Moreover, if $\left\{u_{k}\right\} \subset E$ is a sequence such that $v_{k}=P u_{k} \rightarrow v=P u$ weakly on $N$ and $w_{k}=(I-P) u_{k} \rightarrow w=(I-P) u$ strongly in $M$, where $P$ is the projection of $E$ onto $N$, then $\left\{u_{k}\right\}$ has a renamed subsequence which converges strongly in $L^{2}(\Omega, \rho)$. The reason is that $\left\{v_{k}\right\}$ has such a subsequences because the embedding of $E \ominus N_{0}$ in $L^{2}(\Omega, \rho)$ is compact. Thus $G^{\prime}\left(u_{n}\right) \rightarrow G^{\prime}(u)$ weakly in $E$. Hence all of the hypotheses of Theorem 1.2 are satisfied, and we can conclude that there is a sequence $\left\{u_{k}\right\}$ satisfying (1.13). Write $u_{k}=v_{k}+w_{k}+y_{k}$, where $v_{k} \in N, w_{k} \in M \ominus N_{0}, y_{k} \in N_{0}$. Then

$$
\left(G^{\prime}\left(u_{k}\right), v_{k}\right) / 2=\left([L-\mu] u_{k}, v_{k}\right)-\left(p\left(u_{k}\right), v_{k}\right),
$$

and consequently

$$
\left\|v_{k}\right\|_{E}^{2} \leq\left\|G^{\prime}\left(u_{k}\right)\right\|\left\|v_{k}\right\|_{E} / 2+C\left\|v_{k}\right\|\left(\left\|u_{k}\right\|^{\theta}+1\right)
$$

in view of (3.3) and (3.9). Similarly

$$
\left\|w_{k}\right\|_{E}^{2} \leq\left\|G^{\prime}\left(u_{k}\right)\right\|\left\|w_{k}\right\|_{E} / 2+C\left\|w_{k}\right\|\left(\left\|u_{k}\right\|^{\theta}+1\right) .
$$

If $N_{0}=\{0\}$, then if follows from (3.14) and (3.15) that $\left\|u_{k}\right\|_{E}$ is bounded, and consequently there is a renamed subsequence which converges weakly in $E$ and strongly in $L^{2}(\Omega, \rho)$ to a function $u$. Thus $G^{\prime}\left(u_{k}\right) \rightarrow G^{\prime}(u)$ weakly. But $G^{\prime}\left(u_{k}\right) \rightarrow 0$. Consequently $G^{\prime}(u)=0$, and the proof for this case is complete. If $N_{0} \neq\{0\}$, we note that

$$
\left\|y_{k}\right\|_{E}^{2} \leq\left\|G^{\prime}\left(u_{k}\right)\right\|\left\|y_{k}\right\|_{E} / 2+C\left\|y_{k}\right\|\left(\left\|u_{k}\right\|^{\theta}+1\right)
$$

as well. Again this together with (3.14) and (3.15) implies that $\left\|u_{k}\right\|_{E}$ is bounded and has a renamed subsequence which converges weakly in $E$ and 
such that $u_{k}^{\prime}=v_{k}+w_{k}$ converges strongly in $L^{2}(\Omega, \rho)$. Now

$$
\begin{aligned}
\left(G^{\prime}\left(u_{k}\right), y_{k}-y\right) / 2= & \left([L-\mu]\left(y_{k}-y\right), y_{k}-y\right) \\
& -\left(p\left(u_{k}\right)-p\left(u_{k}^{\prime}+y\right), y_{k}-y\right) \\
& +\left(p\left(u_{k}^{\prime}+y\right)-p(u), y_{k}-y\right) \\
& +\left([L-\mu] y, y_{k}-y\right),
\end{aligned}
$$

where $y_{k} \rightarrow y$ weakly in $E$ and $L^{2}(\Omega, \rho)$ and $u_{k}^{\prime} \rightarrow u^{\prime}$ weakly in $E$ and strongly in $L^{2}(\Omega, \rho)$. By hypothesis

$$
\left(p\left(u_{k}\right)-p\left(u_{k}^{\prime}+y\right), y_{k}-y\right) \geq 0
$$

since $\mu<\lambda_{0}$. Moreover,

$$
\begin{aligned}
\left(G^{\prime}\left(u_{k}\right), y_{k}-y\right) & \rightarrow 0 \\
\left(p\left(u_{k}^{\prime}+y\right)-p(u), y_{k}-y\right) & \rightarrow 0
\end{aligned}
$$

and

$$
\left([L-\mu] y, y_{k}-y\right) \rightarrow 0 .
$$

Hence

$$
\left\|y_{k}-y\right\|_{E}^{2} \leq o(1), \quad k \rightarrow \infty .
$$

This shows that $y_{k} \rightarrow y$ in $E$, and the proof proceeds as before. If $\lambda_{0}<\mu$, we apply Theorem 1.2 to $-G(u)$ and come to the same conclusion. In this case, the inequality in (3.17) is reversed. This completes the proof.

\section{WEAK LINKING}

We now give a proof of Theorem 1.2. It is similar to those of [KS,S2,3, Wi]. Assume that there is no sequence satisfying (1.13). Then there is a positive number $\delta$ such that

$$
\left\|G^{\prime}(u)\right\| \geq 2 \delta
$$

whenever $u$ belongs to the set

$$
E_{1}=\left\{u \in E: b_{0}-2 \delta \leq G(u) \leq a_{1}+2 \delta\right\} .
$$

Since $N$ is separable, we can norm it with a norm $|v|_{w}$ satisfying

$$
|v|_{w} \leq\|v\|, \quad v \in N
$$

and such that the topology induced by this norm is equivalent to the weak topology of $N$ on bounded subsets of $N$ (cf., e.g., [DS,p.426]). For $u \in E$, we write $u=v+w$, where $v \in N, w \in M=N^{\perp}$, and take

$$
|u|_{w}^{2}=|v|_{w}^{2}+\|w\|^{2} \text {. }
$$

Then clearly

$$
|u|_{w} \leq\|u\|, \quad u \in E
$$

and convergence of a bounded sequence $u_{n}=v_{n}+w_{n}$ with respect to this norm means that $v_{n}$ converges weakly in $N$ and $w_{n}$ converges strongly in 
$M$. We denote $E$ equipped with this norm by $E_{w}$. For $u \in E_{1}$, let $q(u)=$ $G^{\prime}(u) /\left\|G^{\prime}(u)\right\|$. Then by $(4.1)$

$$
\left(G^{\prime}(u), q(u)\right) \geq 2 \delta, \quad u \in E_{1} .
$$

Let $T=\left(a_{1}-b_{0}+4 \delta\right) / \delta, R=T+\sup _{Q}\|u\|$, and $B=\bar{B}_{R} \cap E_{1}$, where $B_{R}=\{u \in E:\|u\|<R\}$. For each $u \in B$ there is a $E_{w}$ neighborhood $W(u)$ of $u$ such that

$$
\left(G^{\prime}(h), q(u)\right)>\delta, \quad h \in W(u) \cap B .
$$

For otherwise there would be a sequence $\left\{h_{k}\right\} \subset B$ such that

$$
\left|h_{k}-u\right|_{w} \rightarrow 0 \text { and }\left(G^{\prime}\left(h_{k}\right), q(u)\right) \leq \delta .
$$

Since $B$ is bounded in $E, P h_{k} \rightarrow P u$ weakly in $N$ and $(I-P) h_{k} \rightarrow(I-P) u$ strongly in $M$. Hence, by hypothesis,

$$
\left(G^{\prime}\left(h_{k}\right), q(u)\right) \rightarrow\left(G^{\prime}(u), q(u)\right) \geq 2 \delta
$$

in view of (4.6). This contradicts (4.8). Let $B_{w}$ be the set $B$ with the inherited topology of $E_{w}$. It is a metric space, and $W(u) \cap B$ is an open set in this space. Thus $\{W(u) \cap B\}, u \in B$, is an open covering of the paracompact space $B_{w}$. Consequently, there is a locally finite refinement $\left\{W_{\tau}\right\}$ of this cover. For each $\tau$ there is an element $u_{\tau}$ such that $W_{\tau} \subset W\left(u_{\tau}\right)$. Let $\left\{\psi_{\tau}\right\}$ be a partition of unity subordinate to this covering. Each $\psi_{\tau}$ is locally Lipschitz continuous with respect to the norm $|u|_{w}$ and consequently with respect to the norm of $E$. Let

$$
Y(u)=\sum \psi_{\tau}(u) q\left(u_{\tau}\right), \quad u \in B .
$$

Then $Y(u)$ is locally Lipschitz continuous with respect to both norms. Moreover,

$$
\|Y(u)\| \leq \sum \psi_{\tau}(u)\left\|q\left(u_{\tau}\right)\right\| \leq 1
$$

and

$$
\left(G^{\prime}(u), Y(u)\right)=\sum \psi_{\tau}(u)\left(G^{\prime}(u), h\left(u_{\tau}\right)\right) \geq \delta, \quad u \in B .
$$

For $u \in \bar{Q} \cap E_{1}$, let $\sigma(t) u$ be the solution of

$$
\sigma^{\prime}(t)=-Y(\sigma(t)), \quad t \geq 0, \sigma(0)=u .
$$

Note that $\sigma(t) u$ will exist as long as $\sigma(t) u$ is in $B$. Moreover, it is continuous in $(u, t)$ with respect to both topologies.

Next we note that if $u \in \bar{Q} \cap E_{1}$ and $\sigma(t) u \in B$, then

$$
\begin{aligned}
d G(\sigma(t) u) / d t & =\left(G^{\prime}(\sigma), \sigma^{\prime}\right) \\
& =-\left(G^{\prime}(\sigma), Y(\sigma)\right) \leq-\delta .
\end{aligned}
$$

Hence if $\sigma(t) u \in B$ for $0 \leq t \leq T$, then

$$
G(\sigma(T) u) \leq G(u)-\delta T \leq b_{0}-4 \delta .
$$

But if $\sigma(s) u$ exists for $0 \leq s \leq t<T$, then $\sigma(t) u \in B$. To see this note that

$$
u-\sigma(t) u=z_{t}(u):=\int_{0}^{t} Y(\sigma(s) u) d s .
$$


By $(4.10)$

$$
\left\|z_{t}(u)\right\| \leq t
$$

Consequently

$$
\|\sigma(t) u\| \leq\|u\|+t<R .
$$

Thus $\sigma(t) u \in B$. We can now conclude that for each $u \in \bar{Q} \cap E_{1}$ there is a $t<T$ such that $\sigma(s) u$ exists for $0 \leq s \leq t$ and $G(\sigma(t) u) \leq b_{0}-\delta$. Let

$$
T_{u}:=\inf \left\{t \geq 0: G(\sigma(t) u) \leq b_{0}-\delta\right\}, u \in \bar{Q} \cap E_{1} .
$$

Then $\sigma(t) u$ exists for $0 \leq t \leq T_{u}<T$. Moreover, $T_{u}$ is continuous in $u$. Define

$$
\begin{aligned}
\sigma_{1}(t) u & =\sigma(t) u, \quad 0 \leq t \leq T_{u} \\
& =\sigma\left(T_{u}\right) u, \quad T_{u} \leq t \leq T
\end{aligned}
$$

for $u \in \bar{Q} \cap E_{1}$. For $u \in \bar{Q} \backslash E_{1}$, define $\sigma_{1}(t) u=u, \quad 0 \leq t \leq T$. Then $\sigma_{1}(t) u$ is continuous in $(u, t)$, and

$$
G\left(\sigma_{1}(T) u\right) \leq b_{0}-\delta, \quad u \in \bar{Q} .
$$

Let

$$
\varphi(v, t)=F \sigma_{1}(t) v, \quad v \in \bar{Q}, 0 \leq t \leq T .
$$

Then $\varphi$ is a continuous map of $K:=\bar{Q} \times[0, T]$ to $N$. Moreover, $K$ is a compact subset of $N_{w} \times[0, T]$. For if $\left(v_{k}, t_{k}\right) \in K$, there is a renamed subsequence such that $v_{k} \rightarrow v_{0}$ weakly in $N$ and $t_{k} \rightarrow t_{0}$ in $[0, T]$. Since $\bar{Q}$ is convex, $v_{0}$ is in $\bar{Q}$. Since $\bar{Q}$ is bounded, $\left|v_{k}-v_{0}\right|_{w} \rightarrow 0$. Each $u_{0} \in B$ has a neighborhood $W\left(u_{0}\right)$ in $E_{w}$ and a finite dimensional subspace $S\left(u_{0}\right)$ such that $Y(u) \subset S\left(u_{0}\right)$ for $u \in W\left(u_{0}\right) \cap B$. Since $\sigma_{1}(t) v$ is continuous is $(v, t)$, for each $\left(v_{0}, t_{0}\right)$ in $K$ there are a neighborhood $W\left(v_{0}, t_{0}\right) \subset N \times[0, T]$ and a finite dimensional subspace $S\left(v_{0}, t_{0}\right) \subset N$ such that $F z_{1 t}(v) \subset S\left(v_{0}, t_{0}\right)$ for $(v, t) \in W\left(v_{0}, t_{0}\right)$, where

$$
z_{1 t}(v):=\int_{0}^{t} Y\left(\sigma_{1}(s) v\right) d s .
$$

Since $K$ is compact, there is a finite number of points $\left(v_{j}, t_{j}\right) \subset K$ such that $K \subset W=\cup W\left(v_{j}, t_{j}\right)$. Let $S$ be a finite dimensional subspace of $N$ containing $p$ and all the $S\left(v_{j}, t_{j}\right)$. We note that $\varphi(v, t)$ maps $\bar{Q} \cap S \times[0, T]$ into $S$ since $F \sigma_{1}(t) v=v-F z_{1 t}(v)$, and $F z_{1 t}(v)$ is in $S$ when $v$ is in $\bar{Q}$. Let $\varphi_{t}(v)=\varphi(v, t),(v, t) \in K$. Then

$$
\varphi_{t}(v) \neq p, \quad v \in \partial(Q \cap S)=\partial Q \cap S, 0 \leq t \leq T .
$$

For if $\varphi(v, t)=p$, then $\sigma_{1}(t) v \in F^{-1}(p)=B$. This implies $G\left(\sigma_{1}(t) v\right) \geq b_{0}$ by (1.12). But (4.13) and (1.12) imply that $G\left(\sigma_{1}(t) v\right)<b_{0}$ for $t>0$. Since $p \notin \partial Q$ by hypothesis, $\varphi_{0}(v)=v \neq p$. Thus (4.21) holds. Consequently the Brouwer degree $d\left(\varphi_{t}, Q \cap S, p\right)$ can be defined. Since $\varphi_{t}(v)$ is continuous, we have

$$
d\left(\varphi_{T}, Q \cap S, p\right)=d\left(\varphi_{0}, Q \cap S, p\right)=d(I, Q \cap S, p)=1 .
$$


Hence there is a $v \in Q$ such that $F \sigma_{1}(T) v=p$. Consequently, $\sigma_{1}(T) v \in$ $F^{-1}(p)=B$. In view of $(1.12)$, this implies

$$
G\left(\sigma_{1}(T) u\right) \geq b_{0},
$$

contradicting (4.19). This completes the proof.

\section{REFERENCES}

[BNM1] A. K. Ben-Naoum and J. Mawhin, Periodic solutions of some semilinear wave equations on balls and on spheres, Topol. Methods Nonlinear Anal. 1 (1993), 113-137.

[BNM1] A. K. Ben-Naoum and J. Mawhin, The periodic-Dirichlet problem for some semilinear wave equations, J. Differential Equations, 96 (1992), 340-354.

[DS] N. Dunford and J. T. Schwartz, Linear Operators, Part I, Interscience, 1967.

[KS] W. Kryszewski and A. Szulkin, Generalized linking theorems with an application to semilinear Schrodinger equation, preprint.

[M] J. Mawhin, Nonlinear functional analysis and periodic solution of semilinear wave equation, in "Nonlinear Phenomena in Mathematical Sciences", (ed. V. Lakshmikantham), Academic Press, 1982, 671-681.

[S1] M. Schechter, Infinite dimensional linking, Univ. California Irvine, Math Dept. Preprint Series, \#961116.

[S2] M. Schechter, Weak linking, Univ. California Irvine, Math Dept. Preprint Series, \#970202.

[S3] M. Schechter, Critical point theory with weak-to-weak linking, preprint.

[SM] M. W. Smiley, Time periodic solutions of nonlinear wave equations in balls, in Oscillations, Bifurcation an Chaos (Toronto, 1986), Canad. Math. Soc. Conf. Proc., 1987, 287-297.

[WA] G. Watson, A Treatise on the Theory of Bessel Functions, University Press, Cambridge, 1922 .

[WI] M. Willem, Minimax Theorems, Birkhaüser, 1996.

Department of Mathematics

University of CALIFornia IRVINE

IRVINE, CA 92717, USA

E-mail: mschecht@math.uci.edu 


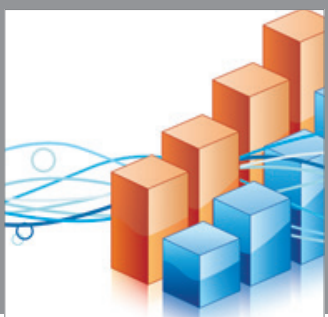

Advances in

Operations Research

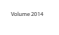

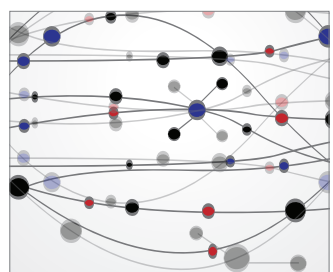

\section{The Scientific} World Journal
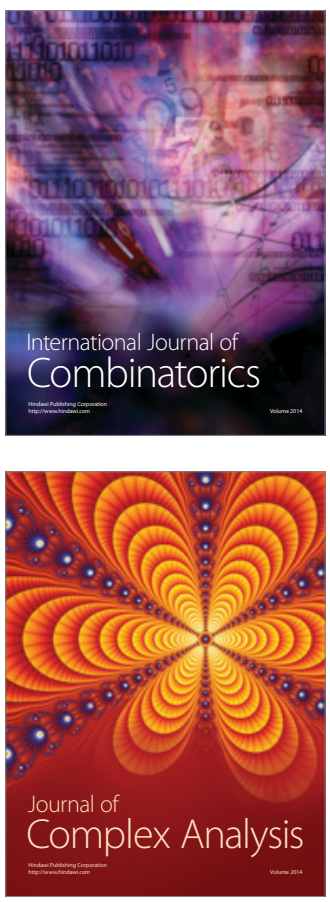

International Journal of

Mathematics and

Mathematical

Sciences
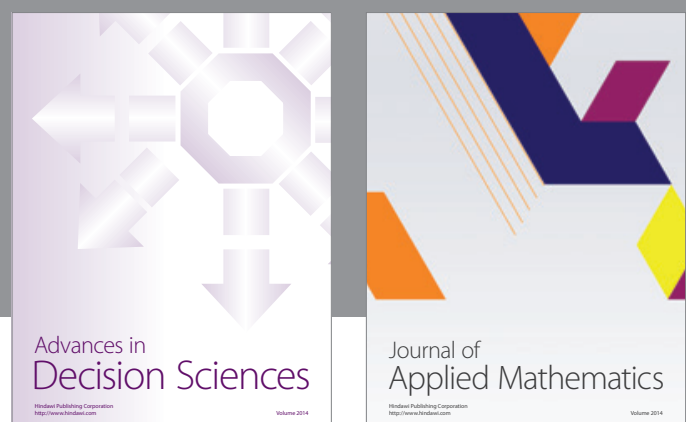

Journal of

Applied Mathematics
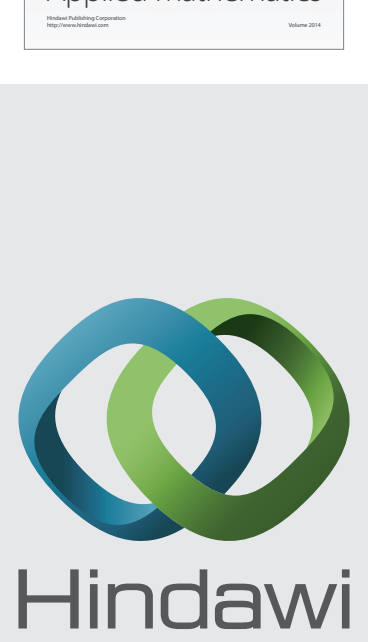

Submit your manuscripts at http://www.hindawi.com
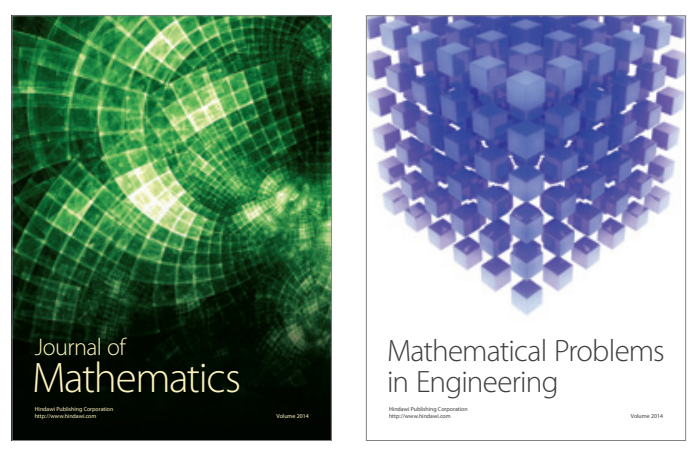

Mathematical Problems in Engineering
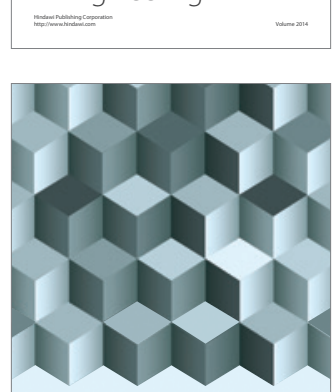

Journal of

Function Spaces
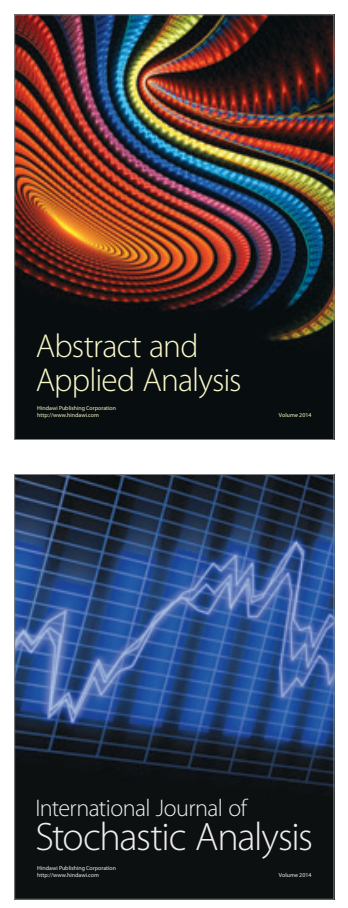

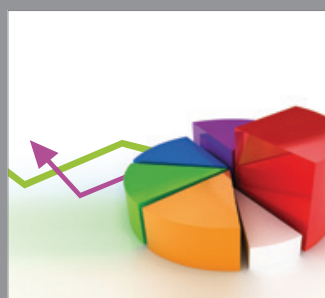

ournal of

Probability and Statistics

Promensencen
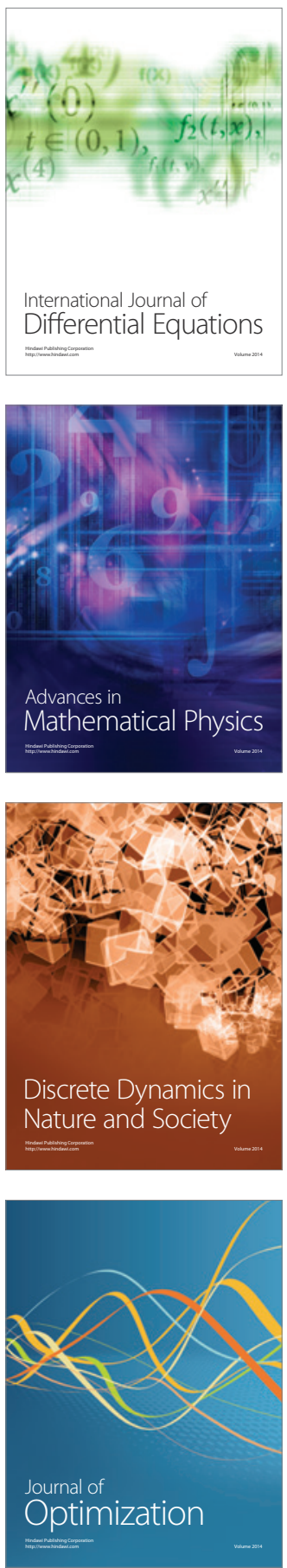